\title{
Fiber bursts as 3D coronal magnetic field probe in postflare loops
}

\author{
H. Aurass, G. Rausche, G. Mann, and A. Hofmann
}

\author{
Astrophysical Institute Potsdam, 14482 Potsdam, Germany \\ e-mail: haurass@aip.de
}

Received 18 October 2004 / Accepted 28 January 2005

\begin{abstract}
Fiber bursts appear in some complex solar radio bursts as a continuum fine structure in the frequency range of $150 \cdots 3000 \mathrm{MHz}$. We present and test a new method to use fiber bursts as a probe of the magnetic field strength and the 3D field structure in postflare loops. Thereby we assume that fiber bursts are driven by whistler waves ascending in the postflare loops which act as magnetic traps for nonthermal flare electrons. For a selected event (1997 April 07) we derive from dynamic radio spectra (Potsdam) and Nançay Radio Heliograph imaging data of fiber bursts the coronal magnetic field strength within the fiber burst source. We compare the fiber burst source positions and field strength estimates with the extrapolated potential magnetic field above the flaring active region NOAA 8027 using SOHO-MDI photospheric field data. The field strength from fiber bursts are within a factor of 0.6 to 1.4 of the field strength of the selected subset of potential field lines and give preference to a 3.5 times Newkirk $(1961$, ApJ, 133, 983) coronal density model within the evolving postflare loops. We find independent proof of the physical significance of considering selected potential field lines as postflare loop field information regarding topology and field strength over a time interval of one hour after the impulsive flare phase. We conclude that radio decimeter and meter wave spectra and radio imaging at two representative frequencies are sufficient for a reliable estimate of the (otherwise not measurable) coronal magnetic field strength in postflare loops. This can be an important field sounding method using the forthcoming FASR (Frequency Agile Solar Radiotelescope) instrument.
\end{abstract}

Key words. Sun: corona - Sun: magnetic fields - Sun: flares - Sun: radio radiation - waves - plasmas

\section{Introduction}

The radio burst emission of the Sun in the meter-decimeter waveband is known to be rather varied (McLean \& Labrum 1985 , for a general reference). It often reveals spectral fine structures (FS) on dynamic spectra, that is, narrowband features and fast temporal changes ${ }^{1}$. To some extent, studying such specific signatures can give precise information about physical processes leading to the observed radio emission, as well as about plasma and magnetic field parameters in the coronal sources. Because of the various FS - like zebra patterns, pulsations, fiber or intermediate drift bursts, and others, see Slottje (1981), the type IV continuum can only be explained by plasma wave mechanisms. In two recent papers (Aurass et al. 2003; Zlotnik et al. 2003) we discussed the origin of zebra stripes and broad band pulsations for a well-observed event. Here we continue to revisit meter and decimeter wave FS by a case study on fiber bursts.

Fiber bursts may allow one to measure the strength of the coronal magnetic field in the radio burst source volume (Kuijpers 1975). From its timing during flares, and from the frequency range of the occurence of fibers (between 150 and $3000 \mathrm{MHz}$ ), we expect, in agreement with Benz \& Mann (1998), that fiber burst sources are situated in the system of postflare loops formed below the erupting prominence and

1 We mean a bandwidth of some percent of the observing frequency, and seconds to subseconds time scale. below the flare current sheet (using the dynamic or two-ribbon flare terminology, see e.g. Priest \& Forbes 2000). This means that fiber bursts supply information about the strength, and - as we will see - in some sense also the topology, of the evolving post flare loop system some minutes after the impulsive flare phase. This information is important for testing some aspects of models of arcade flares (e.g. Martens \& Kuin 1989; Priest $\&$ Forbes 1990) and cannot be obtained by any other means. An important question is how to extract this information from the radio data, and how reliable field strength estimates are obtained this way. Krüger \& Hildebrandt (1993) summarized several principally possible radio methods to determine the field strength in the corona. But currently there is no convincing method ready for application, at least at meter and decimeter wavelengths, i.e. in the lower corona.

The same is true for the dynamically changing coronal field during the impulsive flare phase. Under simplifying conditions (potential or force-free field) the static coronal magnetic field can be extrapolated from given photospherically-measured values (e.g. Seehafer 1978; Sakurai 1982).

We use for our work the sweep spetrograph data of the Astrophysical Institute Potsdam (800-40 MHz) for the spectral identification of fiber bursts. The fiber burst source sites are obtained from the Nançay Multifrequency Heliograph (NRH, The Radio Heliograph Group 1993) data yielding images at five frequencies in the spectrometer band. We will mainly use here the frequencies 410.5, 327, and 236.6 MHz. Radio imaging data 
reveal the radio source sites of fiber bursts in projection on the disc. This can be compared to solar images in other spectral ranges, also to the projection of the extrapolated magnetic field line pattern on the disc.

Fiber bursts are interpreted as the radio signature of whistler waves excited after their coalescence with Langmuir waves in loops with an unstable distribution of nonthermal electrons. This model was proposed by Kuijpers (1975) and modified by Mann et al. (1987, 1989). Bernold \& Treumann (1983) and Treumann et al. (1990) invoked Alfvén solitons to explain fiber bursts. Benz \& Mann (1998) summarized earlier work and compared the existing models with each other. In the present paper we prefer $^{2}$ the whistler wave model. It reflects the main observational facts about fibers: a frequency drift rate between those of beam-driven (type III) and shockdriven (type II) bursts; a duration at a given frequency being less or equal to a second, an instantaneous bandwidth of some percent of the observing frequency, and mostly an absorption gap at the low frequency edge of the emission ridge. In the dynamic spectrum of a fiber burst, the instantaneous frequency difference between the emission ridge and the absorption gap is determined by the whistler wave frequency and by the ponderomotive force. For simplicity, here we consider this parameter only as a measure of the dominant whistler wave frequency in a wave packet as done by Kuijpers (1975).

Knowing that whistler waves propagate dominantly along the magnetic field lines, the radio source sites of fiber bursts at two frequencies determine - together with a coronal density model - a subset of field lines in the extrapolated coronal magnetic field. So we move from projected images on the disc to a true 3D image of the plasma-magnetic field structure in the corona around the fiber burst source volume. Our intention is to compare the coronal field strength estimate obtained by analyzing fiber burst data with the potential field extrapolation. To obtain this independent field information from measurements in the photosphere we use the potential field extrapolation of SOHO-MDI ${ }^{3}$ magnetograms observed at about the time of fiber burst occurence. The extrapolation is obtained in Cartesian coordinates according to Seehafer \& Staude (1980).

Because we observe fiber bursts over a longer time interval (with intermittency of almost $1 \mathrm{~h}$ ) we can determine evolutionary tendencies in the part of the field that hosts the fiber burst sources. Thus, we obtain the rise speed of the postflare loops and the trace of its footpoints in the photosphere. We discuss our findings with respect to measuring coronal magnetic fields, to postflare loop evolution after the impulsive flare phase, and to the understanding of fiber bursts and whistler wave excitation and propagation in the lower corona.

\section{Observations}

For the analysis of fiber bursts we selected the C $6.92 \mathrm{~N}$ flare of 07 April 1997 at 13:55-15:23 UT (called event of interest - EOI - henceforth) occuring at S30E20 which contains for

\footnotetext{
2 Correctly, as it turns out, at least for the given event.

3 Michelson Doppler Imager onboard the Solar and Heliospheric Observatory, Scherrer et al. (1995).
}

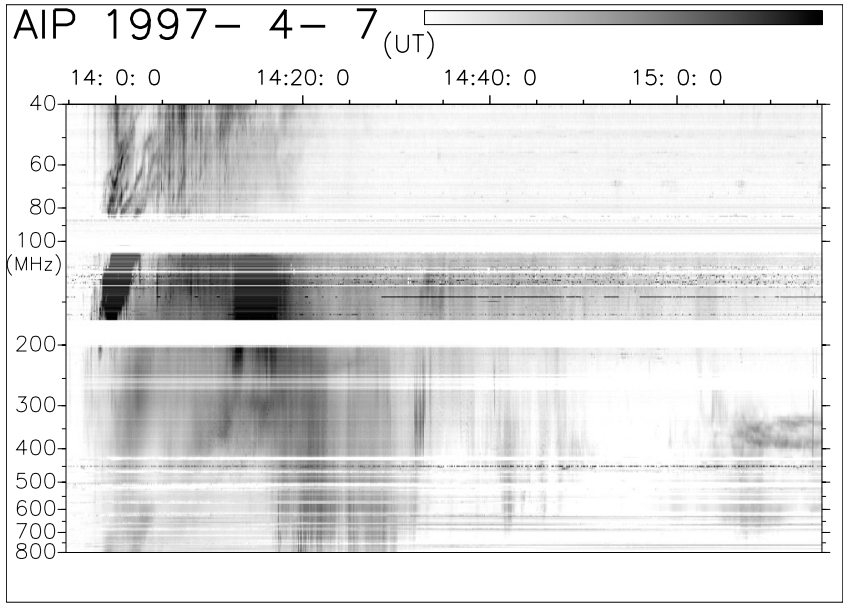

Fig. 1. The dynamic radio spectrum (Astrophysical Institute Potsdam) of the 07 April 1997 flare with a quiet spectrum subtracted. Timeparallel lines are due to local transmitters. The color bar is on the top, right. Left, at low frequencies, the flare-shock wave-excited type II burst with F-H related band-split lanes. Right, at high frequencies, a nondrifting shock signature (see Aurass et al. 2002). The fiber bursts occur between 200 and $600 \mathrm{MHz}$, and are intermittent from 14:07 to 15:05 UT, see Fig. 2.

about $1 \mathrm{~h}$ several patches of continuum emission with fiber burst fine structure. Figure 1 gives the spectrum of the whole event revealing a drifting type II burst, type IV continuum with fine structures, and a late nondrifting type II signature at frequencies above $300 \mathrm{MHz}$.

In Fig. 2a we show single frequency records at the NRH imaging frequencies 327 and $410.5 \mathrm{MHz}$. Enumerated boxes mark fiber burst interval timing. The intervals are listed in Table 1 and contain between 10 and 100 single fiber bursts. Figure $2 b$ gives a fiber burst spectrum sample. Notice the dark, slightly curved emission ridges and the light-gray parallel absorption dips at the low frequency edge. Evolutionary tendencies are evident in overall bandwidth and frequency drift rate. In Fig. 3 we show the fiber frequency drift rate scatter over frequency and time in the whole event. Comparison with the overplotted bold-gray dashed line (after Bernold 1983) reveals that the drift rates in the EOI significantly deviate from Bernold's average curve in the range between 350 and $450 \mathrm{MHz}$. The best coincidence occurs in the intervals 5 and 6, the strongest deviations in the intervals 1,2 and 7. Generally, the fiber burst drift rates decay with time over the event.

The analyzed event is one of the first SOHO-EIT ${ }^{4}$ wave events, and is associated with a halo-CME (Thompson et al. 1999). Yohkoh-SXT ${ }^{5}$ and SOHO-EIT images characterizing the flare and the pre- to postflare reconfiguration of plasmamagnetic field structures in and around AR 8027 were studied by Sterling \& Hudson (1997), Zarro et al. (1999) and Sterling et al. (2000). These authors interpret the EOI as a long duration sigmoid flare and notice the enclosure of postflare EIT loops by

\footnotetext{
4 EUV Imaging Telescope, Delaboudinière et al. (1995).

5 Soft X-ray Telescope onboard the Yohkoh mission, Kosugi et al. (1991).
} 


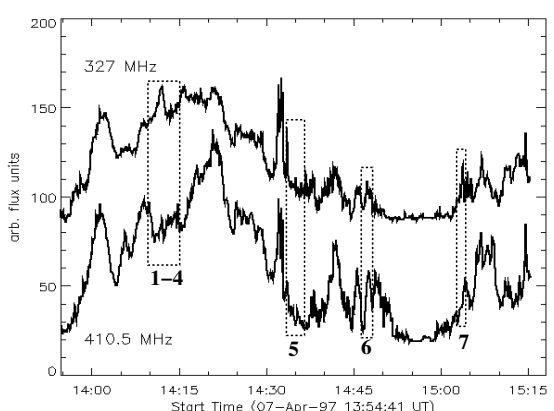

a)

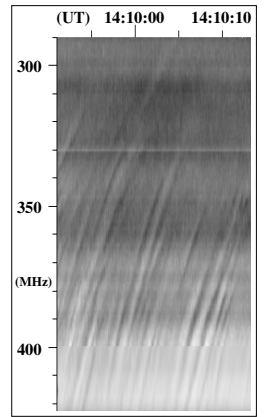

b)

Fig. 2. a) Single frequency records derived from the data shown in Fig. 1 at the NRH frequencies 327 and $410.5 \mathrm{MHz}$. The fiber burst intervals are given by boxes 1-7. b) A typical part of a patch of fiber bursts. Color code as in Fig. 1, no background subtracted.

Table 1. Fiber burst time intervals and parameter range of $\alpha$ yielding common potential field lines crossing the NRH frequency source cubes (compare Figs. 2 and 6a).

\begin{tabular}{cccc}
\hline \hline $\begin{array}{c}\text { Time } \\
\text { interval }\end{array}$ & $\begin{array}{c}\text { Start time } \\
(\mathrm{UT})\end{array}$ & $\begin{array}{c}\text { End time } \\
(\mathrm{UT})\end{array}$ & $\begin{array}{c}\text { Newkirk } \alpha \text {-range } \\
\text { (see Eq. (8)) }\end{array}$ \\
\hline 1 & $14: 09: 32$ & $14: 10: 22$ & $2.5 \ldots 3.75$ \\
2 & $14: 10: 22$ & $14: 11: 22$ & $2.5 \ldots 3.75$ \\
3 & $14: 11: 22$ & $14: 12: 22$ & $\begin{array}{c}\text { no fibers at } \\
\text { NRH frequencies } \\
\text { no fibers at }\end{array}$ \\
4 & $14: 12: 37$ & $14: 14: 07$ & $\begin{array}{c}\text { NRH frequencies } \\
\end{array}$ \\
5 & $14: 33: 02$ & $14: 36: 22$ & $3.0 \ldots 7.5$ \\
6 & $14: 45: 52$ & $14: 46: 42$ & $3.0 \ldots 6.5$ \\
7 & $15: 03: 02$ & $15: 04: 27$ & no fibers at \\
& & & 2 NRH frequencies \\
\hline
\end{tabular}

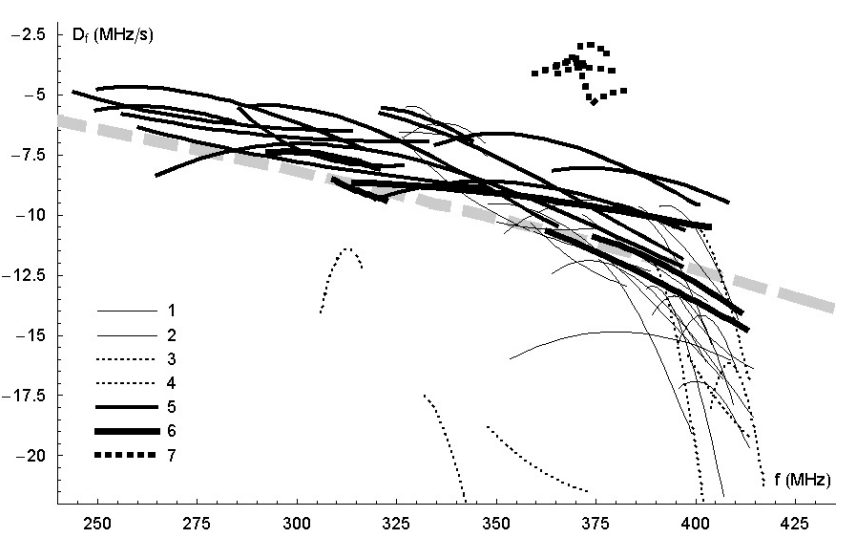

Fig. 3. Fiber burst drift rate evolution over observing frequency and time in the EOI. The bold-gray dashed line is the mean fiber burst drift rate over frequency according to Bernold (1983). For the interval timing see Fig. 2a and Table 1. Note the tendency of falling drift rates over time in the event, as well as the strong deviation from Bernold's mean curve between 350 and $450 \mathrm{MHz}$.

a postflare soft X-ray cusp. Figure 4 shows region NOAA 8027 as part of the SOHO-MDI magnetogram observed at 14:24 UT. Two dominant flux concentrations form a NS-extended neutral line with strong field gradients. Above this line an active region filament is reported which erupts during the flare

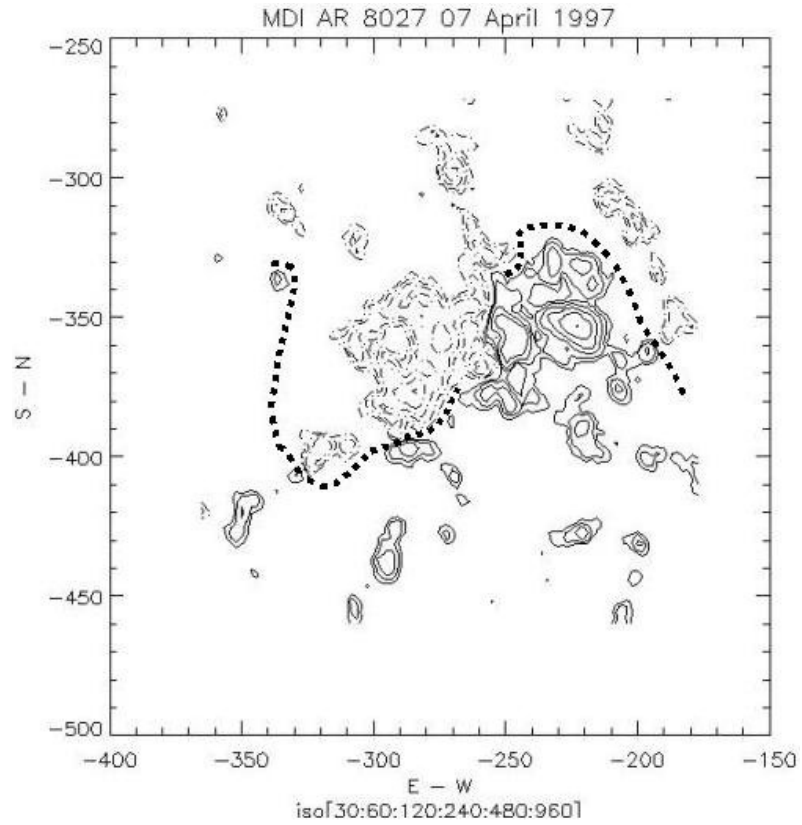

Fig. 4. SOHO-MDI longitudinal field magnetogram of active region NOAA 8027 on 07 April 1997 14:24 UT. Axis in arcsec on the disc, isoline levels in Gauss. The bold dashed line sketches the zero line in the outskirts of the strong field concentrations.

(Zarro et al. 1999). In the southern part of the active region the neutral line is kinked to the SE. In Fig. 4 we show the continuation of the neutral line into the surroundings with distributed fainter flux concentrations. The neutral line well reflects the sigmoidal shape of bright soft X-ray and EIT preflare structures. In the given case, an arcade of low lying loops, simply following the zero line would also appear as a sigmoid if just these loops brighten at the corresponding temperature. We argue that region 8027 can also be classified as a quadrupolar configuration with a NW-SE inclined axis and some shear between the fainter remote (outer) flux concentrations and the two dominant flux sources in between. Sterling \& Moore (2004) discuss a quiescent prominence eruption in a quadrupolar configuration.

The dynamic radio spectrum of the EOI was discussed by Aurass et al. (2002) who indicated the nondrifting type II-like continuum near $300 \mathrm{MHz}$ (starting right in Fig. 1). This feature was interpreted as the radio signature of the lower reconnection outflow termination shock. Figure 5 gives a synopsis of the centers of spectrally distinct radio sources during the EOI overplotted onto a postflare Yohkoh soft X-ray image (courtesy: the Yohkoh home page). The leading main flux concentration in Fig. 4 at (E-225, S-355) is responsible for a dominant concentration of different coronal radio sources (in projection on the disc). It is also the root of the NW branch of the preflare sigmoid and of the leading footpoint of what is called a postflare X-ray cusp structure. The dashed white line between the X-ray-cusp footpoints marks the occurence of the source centroids of the fiber burst sources which are the focus of interest in this paper. North of this dashed line, one recognizes in the X-ray image those postflare loops which will be discussed later with respect to the fiber burst sources. From $\mathrm{H} \alpha$ observations (Kanzelhöhe Observatory, courtesy W. Otruba) 


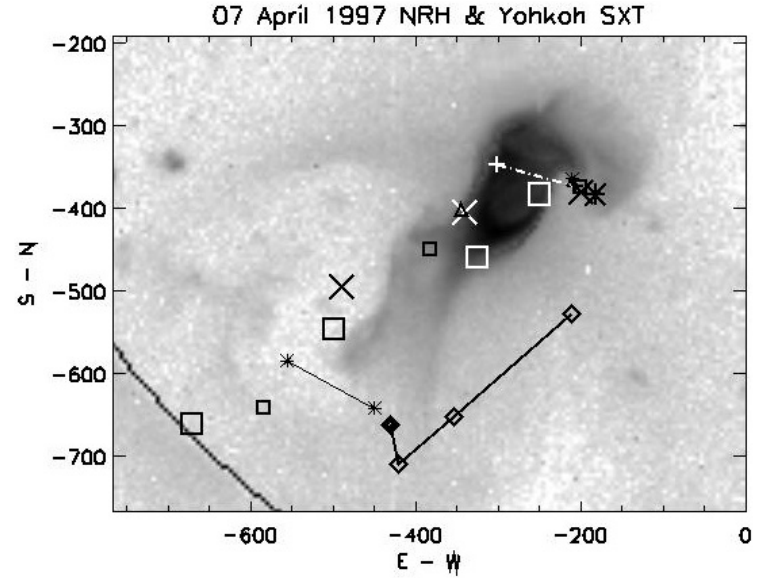

Fig. 5. A Yohkoh postflare soft X-ray image (16:40:28 UT, AlMg filter, $5.4 \mathrm{~s}$ exposure) superposed on the NRH radio source centroids (symbols) during the EOI. Scales in arcseconds. Diamonds: initial type U burst, from $\mathrm{W}$ to $\mathrm{E}$ at 327 , twice at $236.6 \mathrm{MHz}$ and again at $327 \mathrm{MHz}$. Large and small squares: type II and precursor sources at 164 and $236 \mathrm{MHz}$ (13:58-13:59 UT). Small asterisks: correlated sources (327 and $430 \mathrm{MHz}$ ) before the type II burst onset (at <13:58 UT). Triangel: $410 \mathrm{MHz}$ before type II onset. Large asterisk: late source at $327 \mathrm{MHz}$ (see Aurass et al. 2002). Large X: postions given in Sterling \& Hudson (1997), and Zarro et al. (1999), for soft X-ray- and EUV-dimmings. The dashed white line between "+"-source centroids of hundreds of fiber bursts treated in this paper.

we know that the leading flare band moves from the neutral line toward the leading main flux concentration and contacts the included sunspot between 14:07 and 14:11 UT (A. Warmuth, personal communication) just during the occurence of the first fiber bursts. Later the flare band covers the spot region but stops thereafter.

As mentioned, a cluster of different radio sources occurs next to the leading flux concentration in the course of the event. These are one of three type II bursts and one precursor source (large square and small asterisk in Fig. 5), the source of the late nondrifting type II (the termination shock-large asterisk), and some of the fiber burst source centroids ("+"). At the beginning of the event, a faint type $U$ burst (continuous line connecting diamonds) starts due to electron beam injection south of the active region and moves, in projection on the disc, to the top of the (postflare) soft X-ray cusp, where type II burst precursor emission and a further type II burst source also are seen. The starting point of this $U$ burst should be related to the proper flare ignition. A third type II source appears, in projection, between the two mentioned radio source clusters. We have copied in our Fig. 5 as "X" the soft X-ray and EIT dimmings R1, R2, and R3 described by Sterling \& Hudson (1997) and Zarro et al. (1999). Remarkably, in the given case, to each coronal dimming a type II burst radio source appears, occuring already early in the flare. This tells us that at the given sites, early in the flare, there must be comparatively low Alfvén speeds in the active region corona, meaning that here dimming is not only a density decrease but also a relatively more important magnetic field strength decrease! We will return to this point in a forthcoming paper.

\section{Analysis}

\subsection{Some fiber burst theory}

To interpret the intermediate drift bursts we adopt in this paper the whistler wave model of fibers by Kuijpers (1975). In this model the whistler frequency $\omega_{\mathrm{w}}$ is obtained by the instantaneous frequency difference between the emission ridge and the absorption gap in the dynamic spectrum. The theory presumes that the fiber burst pattern is caused by the coalescence of propagating whistler wave packets and Langmuir waves $\left(\omega_{\mathrm{L}}\right)$ to transverse radio waves $\left(\omega_{\mathrm{t}}\right)$ with $\omega_{\mathrm{t}}=\omega_{\mathrm{L}}+\omega_{\mathrm{w}}$. The wave transformation process should lead to a flux increase at $\omega_{\mathrm{pe}}+\omega_{\mathrm{w}}$ and consequently to a flux decrease at the local electron plasma frequency $\omega_{\text {pe }}\left(\omega_{\mathrm{w}} \ll \omega_{\text {pe }}\right)$ where the electron plasma frequency is defined by

$\omega_{\mathrm{pe}}^{2}=\frac{N_{\mathrm{e}} e^{2}}{\epsilon_{0} m_{\mathrm{e}}}$

$\left(N_{\mathrm{e}}-\right.$ electron number density, $m_{\mathrm{e}}-$ electron mass, $\epsilon_{0}-$ dielectric constant, $e$ - elementary charge). Indeed, the absorption gap is generally but not always observed.

The presence of whistler waves in coronal loops may be due to a loss cone instability of trapped high energy electrons (Kuijpers 1975). In correspondence with our earlier work (Aurass et al. 1999, 2002) we consider magnetically asymmetric loops as the probable trap configuration of type IV radio burst fine structures. The loss cone instability grows in the weak-field-footpoint branch of the trap because particles reach higher densities there before being magnetically mirrored. In this branch we expect to find the ascending whistler wave packets and the corresponding fiber burst radio sources exciting negatively drifting spectral signatures.

Because the instantaneous absorption gap - emission ridge separation is assumed to be equal to the dominant whistler frequency of the propagating wave packet, the local coronal magnetic field strength $B$ is most directly determined by this observable (Kujpers 1975). The whistler wave group velocity

$v_{\mathrm{g}}=2 c \frac{\omega_{\mathrm{ce}}}{\omega_{\mathrm{pe}}} \sqrt{x(x-1)^{3}}$

follows from the whistler wave dispersion relation (Kuijpers 1975) with

$x=\frac{\omega_{\mathrm{w}}}{\omega_{\mathrm{ce}}}=\frac{m_{\mathrm{e}}}{e} \frac{\omega_{\mathrm{w}}}{B}$

$\left(\omega_{\mathrm{w}}-\right.$ whistler frequency, $\omega_{\mathrm{ce}}-$ electron cyclotron frequency, $c$ - speed of light). The relation between the whistler group velocity $v_{\mathrm{g}}$ and the radial speed $v_{\mathrm{r}}$ of the radio source

$v_{\mathrm{r}}=v_{\mathrm{g}} \cos \theta$

$(\theta$ - inclination between fiber burst guiding field line and the electron density gradient) together with the fiber burst frequency drift rate

$D_{\mathrm{f}}=\frac{1}{2 \pi} \frac{\mathrm{d}}{\mathrm{d} t} \omega_{\mathrm{pe}}\left(N_{\mathrm{e}}(r(t))\right)=\frac{\omega_{\mathrm{pe}}}{4 \pi} \frac{1}{N_{\mathrm{e}}} \frac{\mathrm{d} N_{\mathrm{e}}}{\mathrm{d} r} v_{\mathrm{r}}$ 
yields

$x(x-1)^{3}=\left(\frac{2 \pi m_{\mathrm{e}}}{c e}\right)^{2}\left(\frac{\lambda_{\mathrm{r}} D_{\mathrm{f}}}{B \cos \theta}\right)^{2}$.

Thereby

$\lambda_{\mathrm{r}}=\left(\frac{1}{N_{\mathrm{e}}} \frac{\mathrm{d} N_{\mathrm{e}}}{\mathrm{d} r}\right)^{-1}$

is the local height scale. For our investigation we used the Newkirk (1961) model

$N_{\mathrm{e}}=\alpha N_{0} 10^{4.32 \cdot R_{\mathrm{s}} / r}$

( $N_{0}=4.2 \times 10^{10} \mathrm{~m}^{-3}, R_{\mathrm{S}}$ - solar radius, $r$ - distance to the solar centre). It corresponds to a barometric density model with a typical coronal temperature of $T=1.4 \times 10^{6} \mathrm{~K}$ (Mann et al. 1999). According to our experience we expect a value of $\alpha=$ $3 . . .4$ for the density model parameter.

\subsection{Data analysis method}

Here, we proceed in two steps as follows:

1. Whistler waves propagate dominantly along the magnetic field lines (e.g. Kuijpers 1975). If whistler wave packets become radio visible, the source site should trace those regions of the magnetic field that guide the whistler wave propagation. Plasma emission is excited near the local plasma frequency which depends on the electron density according to (1). A coronal density model determines the (radial) radio source height. Together with the source coordinates in projection on the disc derived from the NRH imaging data we obtain (at least for the NRH frequencies) the 3D information about the fiber burst source position. There, an estimate of the field strength from fiber bursts can be compared with the strength of the extrapolated potential field at the same point given a known density model.

2. If a fiber burst passes two neighboring NRH frequencies we demand that the corresponding source sites are uniquely connected by the same magnetic field line. Having the information at two frequencies (two heights), and respecting the $2 \mathrm{D}$ source size (given by the projected extent) times a height given by the spatial sampling of the potential field line data ${ }^{6}$, a small subset of "fiber burst field lines" is determined. Vice versa, assuming correct fiber burst theory, and assuming that the potential field is a good guess for the true coronal magnetic field, we can fit both field values by varying the density model (the $\alpha$-parameter in Eq. (8)).

In comparison with Kuijpers (1975) we combine his two methods of field strength determination avoiding the explicite use of the parameter $x$ (inserting Eq. (3) into Eq. (6)), we include the field strength and topological information from the potential field extrapolation, and we apply the radio source localization data.

${ }^{6}$ Which is larger than the height resolution due to the receiver bandwidth of the NRH.
The dynamic spectrum of the EOI contains several fiber burst clusters in the frequency range $200 \mathrm{MHz}$ to $450 \mathrm{MHz}$ (Fig. 2). From these clusters we selected time intervals of $50 \ldots .200 \mathrm{~s}$ duration. We demanded that as much as possible fiber bursts of similar drift rate intersect at least two neighboring NRH frequencies.

For a subset of fiber bursts ${ }^{7}$ in the time intervals, we fitted the burst trajectories by third order polynomials (Fig. 6b). This results in a set of fiber pattern functions $f=F(t)$. Its time derivative gives the fiber frequency drift rate $D_{\mathrm{f}}(t)=\mathrm{d} F(t) / \mathrm{d} t$. If we eliminate the parameter $t$ using $t=F^{-1}(f)$, it follows the drift rate $D_{\mathrm{f}}(f)$ as a function of frequency (see Fig. 3).

The fiber pattern function defines the transit time of a burst at a given observing frequency. The cut through the dynamic spectrum for this time yields the instantaneous emission topabsorption gap frequency separation which corresponds to the whistler frequency as explained in Sect. 3.1. Together with the local density height scale (7) all quantities are known to compute the coronal magnetic field strength at the fiber burst source site according to Eqs. (6) and (3).

From the instantaneous absorption-emission separation in the dynamic spectrum, we obtain a minimum and maximum whistler frequency at the NRH observing frequencies within each analyzed time interval. According to Eq. (6) it follows a minimum and a maximum field strength value. These "error bars" will be used later (see Fig. 7). So far we have finished step 1 of the data analysis.

In step 2 of our procedure, we compare the spatial information derived from fiber burst sources with the structure of the extrapolated photospheric magnetic field. We search for "fiber burst field lines" which are associated with the observed radio sources. Using Eqs. (1) and (8) we calculate the (radial) radio source height. The NRH fiber burst data specifying the source projection on the solar disc complete the spatial definition of the radio source.

The analysis of the radio source sites in the fiber burst intervals yields source cubes as shown in Fig. 6a. The cubes are defined as follows: for a selected NRH observing frequency (height level) all fiber bursts passing that level and forming a source during the given time interval remain within that cube. Next, we search for those subsets of field lines of the potential field which penetrate the cubes of a given time interval at two different frequencies (within two neighboring height levels). The cube height is chosen somewhat larger than the arc length element (1.9 arcsec), so that at least one point of an intersecting field line belongs to the cube volume. The choice of $\alpha$ in the density model (8) influences the field line selection because it changes the radio source height.

We assume that $n$ field lines were selected. Now, we represent the field lines using steps of equal arc length $\Delta s(s=$ $\left.0 \ldots s_{\max }(k)\right): \boldsymbol{B}_{k}(s)(k=1 \ldots n)$. From this, a mean "fiber burst field line" is computed after resampling all field lines to a

\footnotetext{
7 The fiber pattern is contrast-enhanced using the time derivative of the dynamic spectrum.
} 

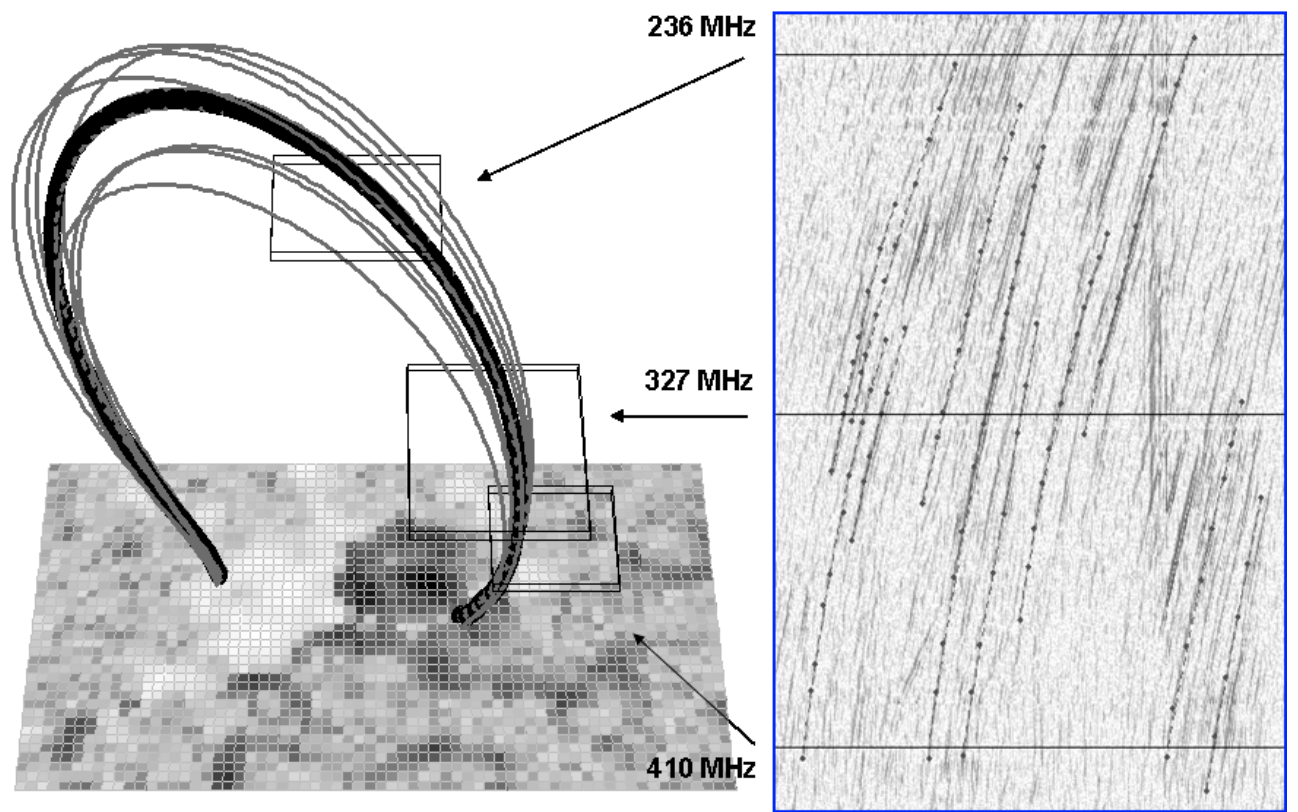

a)

b)

Fig. 6. a) Perspective view of roughly the same part of the MDI magnetogram (N-upwards, W-right) as shown in Fig. 4 with overplotted "fiber burst field lines". The field lines penetrate the source site cubes (boxes) which were constructed from NRH data. The bold lane is a computed "mean field line". Data are from time interval 5, field lines evaluated with the Newkirk $\alpha=3.5$ density model. b) a $60 \mathrm{~s}$ interval (horizontal box length) of the time-derivated AIP dynamic spectrum (gray coded). Overplotted are fitted fibers (dark curves with dots). The arrows point from the NRH observing frequency level (labeled dark lines parallel to the time axis) to the corresponding source site box in a).

normalized length according to $\tilde{s}=s / s_{\max }$. We obtain for the mean field strength

$\boldsymbol{B}_{\text {mean }}(\tilde{s})=\frac{1}{n} \sum_{k} \boldsymbol{B}_{k}(\tilde{s})$

In the same sense it follows for the coordinates of the mean field line sample points

$\boldsymbol{r}_{\text {mean }}(\tilde{s})=\frac{1}{n} \sum_{k} \boldsymbol{r}_{k}(\tilde{s})$.

As an example of a mean field line we give in Fig. 6a a perspective view of the selected field lines above the photospheric plane (sampling drawn as square pattern), the mean field line according to Eq. (10), and for illustration also the radio source cubes derived from the NRH 410.5, 327, and 236.6 MHz imaging data together with a density model (8) with $\alpha=3.5$. Figure 7 shows the mean field strength along the "mean potential" field line, and the field strength from fiber burst drift rates plotted over the Newkirk parameter $\alpha$. As expected, the mean field strength decreases with growing $\alpha$ because the radio source region (and consequently the analyzed height range in the potential field) reaches larger coronal heights. According to the whistler model of fiber burst formation (Sect. 3.1) the observed negative frequency drift rate represents an ascending wave packet travelling at the whistler group velocity along field lines. However, in the dynamic spectrum only the speed component along the density gradient results in a frequency drift. The density model (8) implies a radial density gradient. The radio source sites within the plot of potential field lines reveal the spatial run of the fiber burst-carrying field lines. Thus,

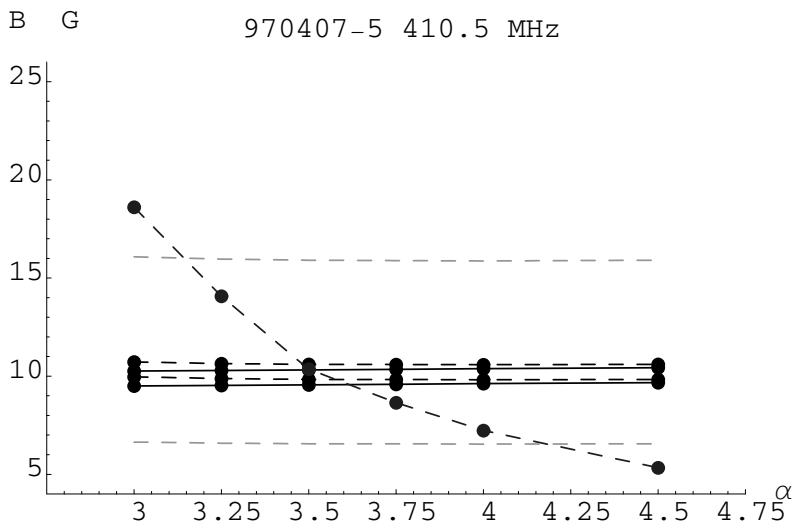

Fig. 7. Comparison of the magnetic field strengths over Newkirk $\alpha$ for time interval 5 and the frequency $410.5 \mathrm{MHz}$. The thick continuous line (after step 1): min. and max. field strength from fiber burst data. Dashed parallel lines: the same, corrected for the inclination $\theta$ between the field line and the density gradient. Gray dashed: 1.5 times corrected maximum field and 0.67 times corrected minimum field strength. Dashed declining curve (after step 2): mean "potential" field line.

the frequency drift rate obtained from the dynamic spectrum can be corrected (according to Benz \& Mann 1998) with the now available spatial information. We obtain the corrected value for the whistler group velocity from Eq. (4). Figure 7 gives the corrected magnetic field values, too (dashed lines). The effect becomes particularly large if a selected field line bundle cuts the NRH source cube near its summit. 


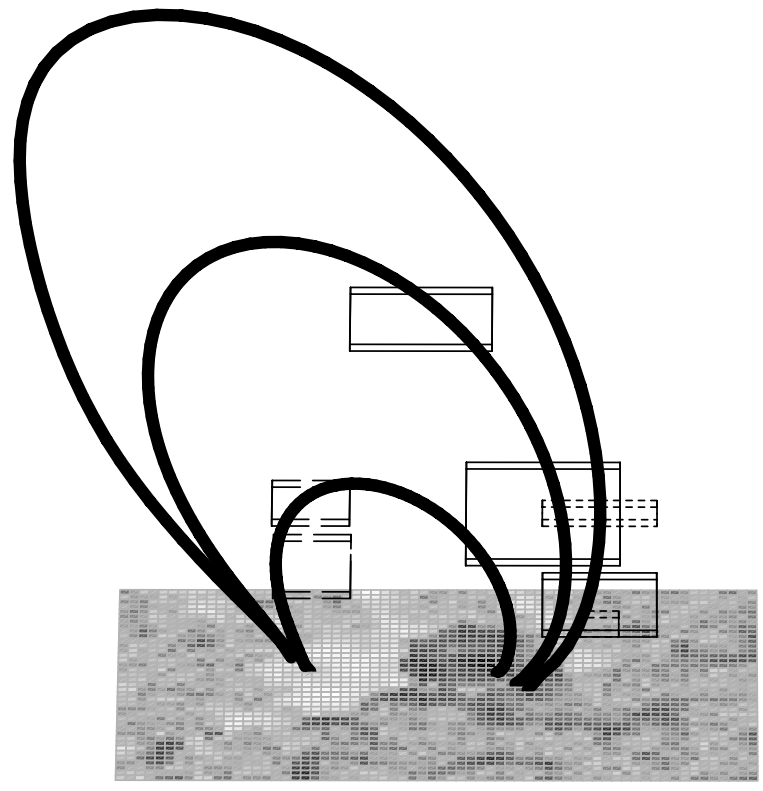

a)

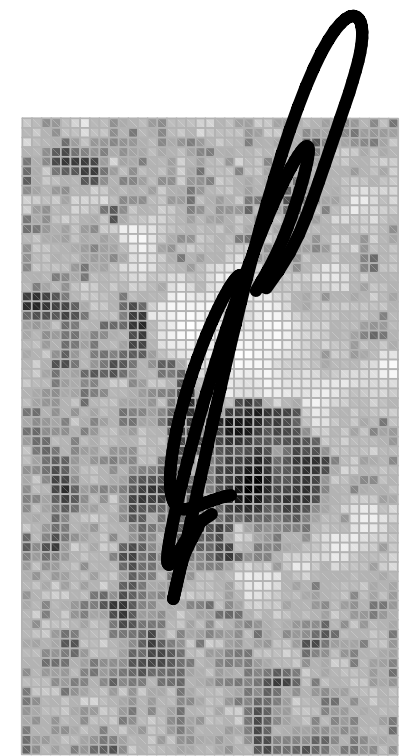

b)

Fig. 8. Perspective views of mean field lines and radio source cubes for the density model (8) with $\alpha=3.5$. a) Side view (N-upwards, W-to the right); b) top view, rotated by $-\pi / 2$. Lowest field line-interval 1 and 2 , middle line-5, upper line- 6 . The lines are asymmetric toward NE. Notice the changing box positions: the fiber sources are situated in the trailing field line branch for intervals 1 and 2 (dashed boxes) in contrast with being placed in the leading branch for intervals 5 (continuous boxes) and 6 (short-dashed boxes).

\subsection{Application}

As already shown in Fig. 3 we started with a statistical analysis of the fiber burst drift rates. We notice a tremendous change in the drift rates during the event and a certain evolutionary tendency. Because of this result we divided the fiber patches into the intervals given in Table 1 and Fig. 2.

The time intervals $1,2,5$, and 6 contain fiber bursts that cut two NRH frequencies. For these intervals we found extrapolated field lines intersecting all frequency levels for a Newkirk $\alpha$ range shown in Table 1 . We used an increment of 0.25 for $\alpha$. The time intervals 1 and 2 were treated together because they are contiguous and the search for field lines yields the same line selection ${ }^{8}$. The fiber bursts in interval 5 pass three of the NRH frequencies $(410.5,327.0,236.6 \mathrm{MHz})$, in the intervals 1,2 , and 6 they pass only two of them $(410.5,327.0 \mathrm{MHz})$ (see Fig. 8a). As mentioned in Table 1 in the remaining intervals no bursts occur at more than one NRH imaging frequency. Therefore no line selection was possible.

The field strength comparison (whistler model and potential field extrapolation) as exemplified in Fig. 7 restricts the range of the parameter $\alpha$. If the field strength along the mean field line is between the gray-dashed lines in Fig. 7, the $\alpha$-range in Table 2 follows. For intervals 5 and 6 the investigations were restricted to $\alpha<4.5$.

In all examined time intervals the value $\alpha=3.5$ best matches the field strength. The computed mean field lines shown in Fig. 8a were selected for this parameter value of the density model (8). Note the change of the radio source

\footnotetext{
8 The interval 2 radio source cubes are insignificantly larger than those of interval 1 , and interval 1 cubes are enclosed in interval 2 cubes.
}

Table 2. The range of parameter $\alpha$ obtained by comparing the field strength from whistler model and potential field. For the given $\alpha$-range the field strength ratio is in $[0.6,1.5]$.

\begin{tabular}{ccc}
\hline \hline Time interval & Frequency $(\mathrm{MHz})$ & $\alpha$-range \\
\hline 1 and 2 & 410.5 & $3.4 \ldots 3.75$ \\
& 327.0 & $2.9 \ldots 3.5$ \\
5 & 236.6 & $2.5 \ldots 3.5$ \\
5 & 410.5 & $3.1 \ldots 4.2$ \\
6 & 327.0 & $3.0 \ldots 4.0$ \\
& 410.5 & $3.3 \ldots 4.5$ \\
& 327.0 & $3.0 \ldots 4.0$ \\
\hline
\end{tabular}

box site from intervals 1 and 2 to intervals 5 and 6 . A check of the corresponding footpoint field strengths reveals that the fiber burst sources always occur in the weak field branch of the mean field lines (compare also the data in Table 3). A rising turning point of the mean field lines is clearly visible with increasing time. The vertical component of the loop summit speed is $(50 \pm 12) \mathrm{km} \mathrm{s}^{-1}$ from intervals 1 and 2 to 5 and $(99 \pm 33) \mathrm{km} \mathrm{s}^{-1}$ from interval 5 to $6^{9}$. In Fig. 8 b a top view of Fig. 8a is displayed. Here, the resulting speed of the projected field line footpoint motion can be determined between the time intervals with fiber bursts. On the eastern side, a speed of $(5.3 \pm 1.5) \mathrm{km} \mathrm{s}^{-1}$ for intervals 1 and 2 to 5 and $(3.4 \pm 2.3) \mathrm{km} \mathrm{s}^{-1}$ for intervals 5 to 6 is found. On the western side it is $(5.3 \pm 1.8) \mathrm{km} \mathrm{s}^{-1}$ for intervals 1 and 2 to 5 and $(7.4 \pm 3.8) \mathrm{km} \mathrm{s}^{-1}$ for intervals 5 to 6 . This leads to an effective footpoint broadening with $(8.1 \pm 2.4) \mathrm{km} \mathrm{s}^{-1}$ (from interval 1 and 2 to 5$)$ and $(6.8 \pm 3.9) \mathrm{km} \mathrm{s}^{-1}$ (from interval 5 to 6 ). Note that all three mean field lines are almost in the same plane, the

\footnotetext{
9 Time taken in the center of the intervals.
} 
Table 3. Parameters of the selected "fiber burst" potential field lines.

\begin{tabular}{cccccc}
\hline \hline Time interval & $B($ East $)(\mathrm{G})$ & $B($ West $)(\mathrm{G})$ & $B_{\min }(\mathrm{G})$ & $h_{\text {top }}(\mathrm{Mm})$ & Footpoint span $(\mathrm{Mm})$ \\
\hline 1 and 2 & 71.4 & 347.0 & 2.9 & 65.8 & 53.2 \\
& 32.7 & 304.2 & 2.5 & 70.2 & 53.5 \\
& 23.1 & 335.2 & 2.5 & 70.5 & 53.5 \\
& 30.9 & 344.5 & 2.4 & 71.6 & 53.6 \\
& 48.3 & 299.3 & 2.7 & 68.2 & 51.9 \\
Mean 1 and 2 & 38.2 & 394.7 & 2.7 & 68.7 & 51.9 \\
5 & 44.8 & 348.9 & 2.9 & 66.4 & 51.8 \\
& $\mathbf{4 1 . 4} \mathbf{1 5 . 8}$ & $\mathbf{3 3 9 . 1} \pm \mathbf{3 1 . 9}$ & $\mathbf{2 . 7} \pm \mathbf{0 . 9}$ & $\mathbf{6 8 . 8} \pm \mathbf{2 . 1}$ & $\mathbf{5 2 . 8} \pm \mathbf{0 . 9}$ \\
& 125.3 & 80.5 & 0.27 & 151.5 & 68.4 \\
& 79.7 & 114.7 & 0.36 & 140.0 & 62.3 \\
& 92.1 & 155.1 & 0.47 & 129.6 & 61.7 \\
& 116.9 & 231.9 & 0.34 & 144.1 & 61.0 \\
& 97.1 & 60.2 & 0.45 & 130.7 & 63.9 \\
Mean 5 & 88.0 & 85.3 & 0.35 & 141.9 & 63.3 \\
6 & 93.3 & 23.4 & 0.37 & 139.2 & 65.5 \\
Mean 6 & 96.3 & 44.7 & 0.29 & 150.4 & 64.8 \\
& 120.3 & 101.2 & 0.44 & 129.7 & 68.0 \\
& 92.5 & 59.8 & 0.28 & 151.5 & 67.3 \\
& $100 . \mathbf{1 5 . 2}$ & $\mathbf{9 5 . 7} \pm \mathbf{6 0 . 7}$ & $\mathbf{0 . 3 6} \pm \mathbf{0 . 0 7}$ & $\mathbf{1 4 0 . 8} \pm \mathbf{8 . 7}$ & $\mathbf{6 4 . 6} \pm \mathbf{2 . 6}$ \\
& 100.5 & 88.4 & 0.06 & 219.7 & 69.2 \\
& & 131.0 & 0.09 & 199.2 & 69.4 \\
& & & & & $\mathbf{6 9 . 3} \pm \mathbf{0 . 1}$ \\
\hline
\end{tabular}

line for intervals 1 and 2 is somewhat inclined to the SE following the general tendency of field lines in the potential field in this range.

\subsection{Summary: Results of data analysis}

Fiber bursts are a type IV radio burst fine structure understood as a signature of whistler wave propagation along postflare loop field lines. The EOI radio spectrum offers a sequence of seven fiber burst patches along the time axis within $1 \mathrm{~h}$. We consider three selected intervals in detail. By application of a new method of determination of the strength of the coronal magnetic field - joining spectral and imaging meter wave radio observations of fiber bursts with photospheric magnetograms and potential field extrapolation into the corona - we obtained the following results:

- The method selects a small but generally dense subset of potential field lines for each fiber burst patch.

- The magnetic field strength ratio between fiber burstderived and potential field line-derived estimate scatters between 0.6 and 1.4 .

- The best (just mentioned) field strength ratio is achieved by using a 3.5 times Newkirk (1961) density model not depending on time in the event.

- For each patch, a mean "potential" fiber burst field line can be calculated.

- The mean field lines are almost coplanar (with about EWorientation) and enclose each other with increasing time.

- We argue that the mean field lines reflect activated ranges of the postflare loop system.

- The leading footpoint of the mean field lines is near the main flare radio source of the event (see Fig. 5), in or next to the leading spot field concentration of AR 8027, and moves out toward lower fields with time.

- The trailing footpoint of the mean field lines is initially next to a local field minimum, and moves out toward higher fields with time.

- Initially the fiber burst sources occur in the trailing branch of the mean field lines. As soon as the trailing footpoint field becomes stronger than the leading footpoint field, the fiber bursts jump to the leading branch of the mean field lines. This means whistler waves leading to fiber burst emission grow in the weak field branch of an asymmetric loop.

- The mean field line summit rises with speeds from 50 to (later) $99 \mathrm{~km} \mathrm{~s}^{-1}$.

- The mean field line footpoints reveal a footpoint motion ${ }^{10}$ of 8 to (later) $7 \mathrm{~km} \mathrm{~s}^{-1}$.

\section{Discussion}

The structure of the magnetic field in the solar corona and its evolution is impressively reflected in the wealth of new space-based observations showing looplike brightenings, magnetic connectivity changes, coronal dimming after eruptions and large-amplitude wave passage, and reconfiguration during prominence eruptions, flares and CMEs. On the other hand, it is still not possible to measure the strength of the magnetic field in the solar corona.

We started this work with the question of whether a reliable estimate of the coronal magnetic field strength can be obtained using a specific radio burst phenomenon called fiber or intermediate drift bursts. Fiber bursts are fine structures in solar type IV

${ }^{10}$ In the sense of growing footpoint distance. 
radio bursts and are understood as a signature of whistler wave propagation along the coronal magnetic field. Combining well known methods in a new manner, we find that the answer is "yes" up to an uncertainty better than a factor of two.

The result is of interest from several aspects: first, it gives a cross-check between two not yet compared procedures to estimate the strength of the coronal magnetic field. These are the potential field extrapolation from photospheric measurements, and a field strength determination based on the whistler wave theory to interpret fiber bursts. Next, we obtain the magnetic field and density information during the ongoing flare process, more precisely out of evolving postflare loops filled with trapped flare-accelerated electrons being the energy source of whistler wave excitation and plasma wave emission. Third, because the result gives topological information about the activated field structures, we can independently compare the selected field pattern with imaging data obtained in other spectral ranges which may well reflect coronal plasma-magnetic field configurations (e.g. Yohkoh soft X-ray images, SOHO EUV images from plasma at coronal temperatures).

The presented analysis selects a specific "activated" part of the plasma-magnetic field structures of the flaring active region - summarized as "mean fiber burst field lines". We will discuss the properties of this selected range in NOAA AR 8027 under the following viewpoints:

1. the properties of fiber burst sources;

2. the spatial association with coronal plasma structures seen in soft X-ray and EUV wavelengths;

3. the evolution of postflare loops, and possible consequences for the flare mechanism.

\subsection{The properties of fiber burst sources}

For a better understanding of solar flare radio emission the knowledge of the plasma-magnetic field structure hosting the sources is important. There, the nonthermal flare electrons experience conditions able to excite escaping radio emission. We can now give a precise description of the fiber burst sources in the EOI. To this aim we present in Table 3 some parameters of the selected potential field lines. We notice several interesting properties:

- The considered sample of fiber bursts is best compatible with a 3.5 fold Newkirk (1961) density model independent of time in the event.

- The fiber burst drift rate decays with time in the event revealing, on average, falling field strength in the source.

- Fiber bursts occur at field strengths of $6 \ldots 14 \mathrm{G}(410 \mathrm{MHz})$, $3 \ldots 7 \mathrm{G}(327 \mathrm{MHz})$ and $3 \mathrm{G}(236 \mathrm{MHz})$ which occur at heights between 20 and $100 \mathrm{Mm}$. The field strength values well correspond with the data given by Kuijpers (1975, Table III).

- On average over the whole event, the ratio $x$ (Eq. (3)) is obtained ${ }^{11}$ as $0.41(410 \mathrm{MHz}), 0.38(327 \mathrm{MHz})$, and

\footnotetext{
11 We used the mean of the extreme whistler wave frequencies, and the mean of the extreme field strength values in the source box, at each observing frequency.
}

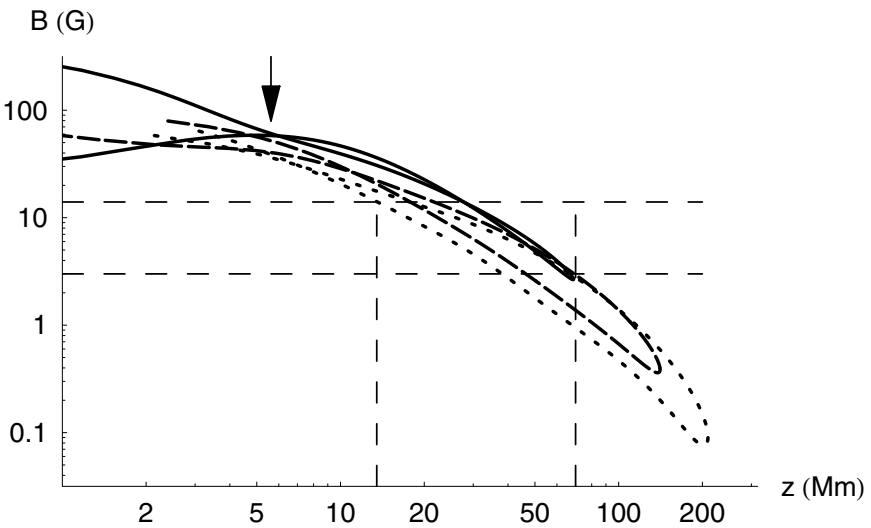

Fig. 9. Field strength along the mean fiber burst field lines versus height over the photosphere. Continuous line: interval 1 and 2, dashed curve: interval 5, dotted curve: interval 6. All fiber bursts occur in the field strength-height range box formed by the long-dashed lines. The arrow points to local field strength maximum occuring near the weak field end of the continuous line at about $5 \mathrm{Mm}$ height.

0.21 (236 MHz). This is well below the limit of whistler wave absorption under coronal conditions (Kuijpers 1975).

- The whistler group velocity scatters around $4000 \mathrm{~km} \mathrm{~s}^{-1}$. The mean ratio of the group velocity of the fiber burst exciter to the Alfvén speed is obtained as $23.2<v_{\mathrm{g}} / v_{\mathrm{A}}<$ 25.7 in the considered frequency range. Consequently, the Alfvén soliton model of Treumann et al. (1990) can be ruled out, at least for the given event.

In intervals 1 and 2 the leading footpoint field strength is almost 10 times stronger than that of the trailing footpoint. In intervals 5 and 6 the mean field strength of the footpoints are less different. The fiber burst source site follows the parameters of the mean field lines ${ }^{12}$ despite some scatter, especially in interval 5. The fibers always occur in those branch of the mean field line that is rooted in smaller fields. Evidently, whistlers are more easily excited there and meet - ascending in this loop branch - the descending trapped electrons, thereby interacting and emitting the escaping radio emission.

Figure 9 gives the height dependence of the magnetic field strength along the mean field lines. The minimum field of interval 6 is about 1 order of magnitude smaller than the minimum field strength in intervals 1 and 2 ; the turning heights behave as 3:1. The minimum field strength does not occur at the turning point of the lines. In intervals 1 and 2 (continuous line), fibers reach almost the top of the loop. Later (interval 5 - dashed, interval 6 - dotted) the burst sources remain in the same height and field range but lower and lower with respect to the loop top. The majority of the selected potential field lines (100\% in intervals 1 and 2, 60\% in interval 5) makes a spatial turn shortly above the weak field footpoint. Following the field strength along the field line it turns out that there is a local maximum in heights of the order of $6 \mathrm{Mm}$ above the photosphere (arrow in Fig. 9). This means bouncing electrons in the trap do not see the true minimum field strength but in the case of intervals 1 and 2 experience about $60 \mathrm{G}$; in case of interval 5

12 This confirms the applied averaging procedure. 


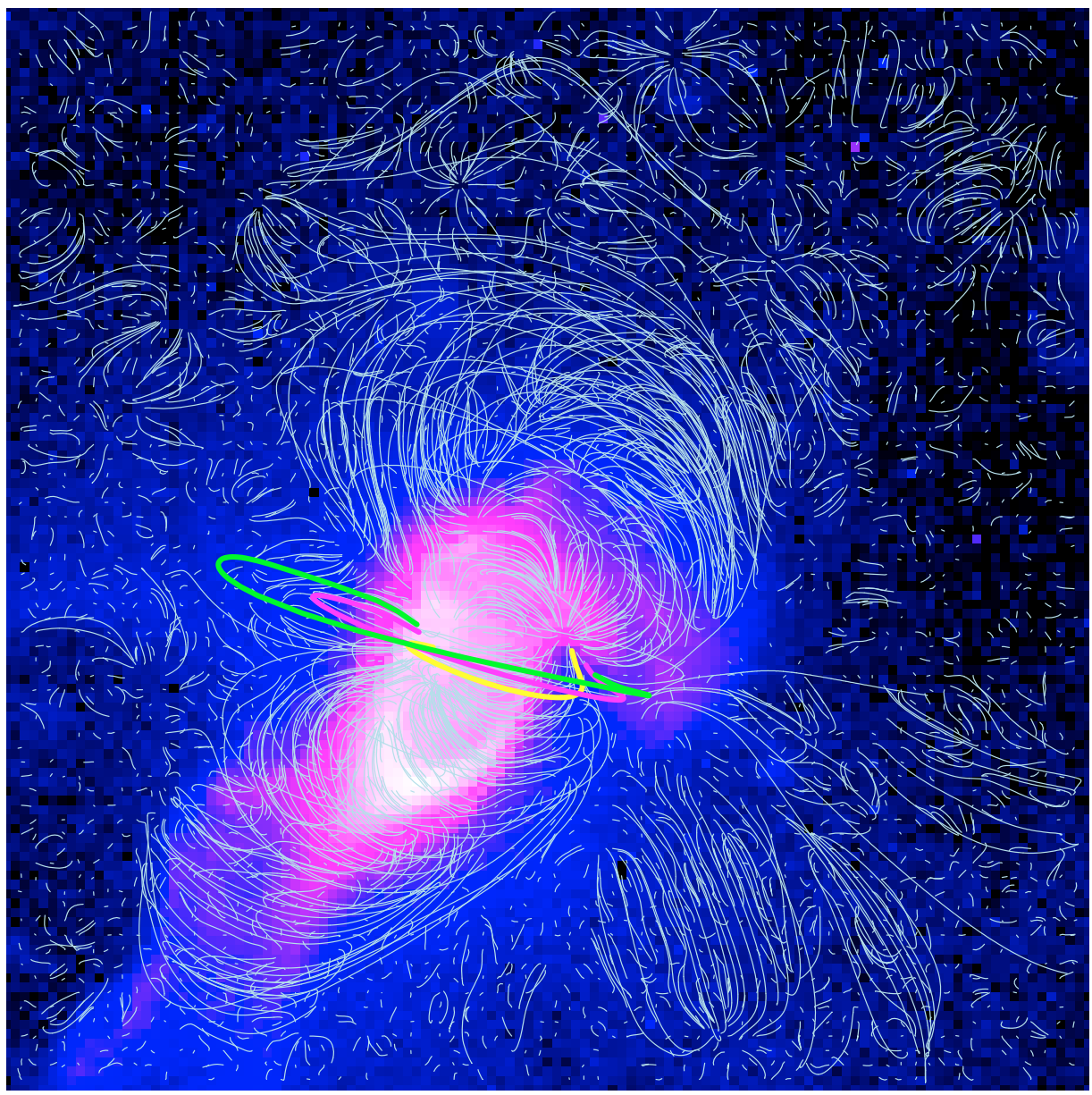

Fig. 10. The Yohkoh postflare SXT image (16:40:28 UT, AlMg filter, 5.4 s exposure, N-upwards, W-to the right) as shown in Fig. 5 with superposed potential field lines and overplotted "mean fiber burst" field lines (from Fig. 8a). Yellow line-intervals 1 and 2, magenta-interval 5, green-interval 6.

about $50 \mathrm{G}$. This property might explain the longer lifetime of the trapped electrons in the radio source-hosting loop structure because electrons are reflected at greater heights with less loss due to collisions, and the characteristic loss cone of the trap is smaller. Such a configuration may be a necessary condition for fiber burst emission.

\subsection{Comparison with soft $X$-ray- and EUV-visible coronal structures}

In Fig. 10 we superposed the grid of all potential field lines over the Yohkoh postflare soft X-ray image. Further, we added our result - the mean fiber burst field lines. The X-ray image and its deviation from the preflare situation is discussed and compared with EUV images from SOHO by Sterling \& Hudson (1997), Zarro et al. (1999), and Sterling et al. (2000). The preand postflare images are dominated by short loops crossing the magnetic neutral line. Low-lying loops seem to form an arcade following the neutral line; a fan of large-altitude connections between the dominating polarity concentrations is best seen in the post- minus preflare difference SXT image shown in Fig. 2c of Sterling \& Hudson (1997). The same loops can also be recognized in our Fig. 5 north of the white dashed line.
The fan also encloses bright soft X-ray loops which are situated in and around the plane of the "mean fiber burst" field lines. The mean field lines are higher than the soft X-ray loops and clearly far from the plane of the highly inclined cusp-like structure. This seems to be in contrast to the bright EUV postflare loops (Sterling \& Hudson 1997; Zarro et al. 1999) which fit in the soft X-ray cusp structure. Knowing our result it is possible that the EUV postflare loops in the plane of the mean field lines are too faint to be recognized on the background of the other bright patterns, or the mean field line-loops are outside of the rather narrow temperature response range of EIT. A more detailed comparison of the X-ray- and EUV images with the extrapolated magnetic field can probably resolve some of the uncertainties but this is beyond the scope of this paper. The mean "potential" fiber burst field lines fit well with a part of the postflare loop fan observed by Yohkoh SXT.

\subsection{Postflare loop evolution in the 07 April 1997 flare event in AR8027}

In the EOI, fiber bursts occur in patches distributed over about $1 \mathrm{~h}$ after the impulsive flare phase. Thus, we can trace the 


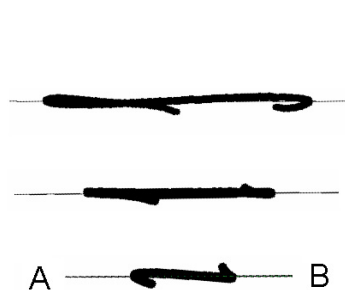

a)

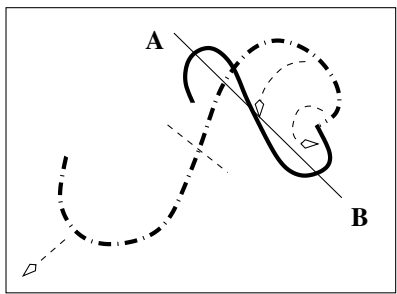

b)
Fig. 11. a) Top view of the least square-deviating plane of the mean field lines (seen edge-on, thin line). From top to bottom interval 6 to intervals 1 and 2. Vertical arrangement arbitrary, relative scale correct. Notice the inverse sigmoid orientation especially in intervals 1 and 2. b) Non-scaled sketch explaining the different winding senses of observed sigmoids: preflare in soft X-rays (dash-dotted, see e.g. Sterling et al. 2000) and the postflare fiber burst-detected mean "potential" field line in intervals 1 and 2 seen edge-on as in a). The arrow pointing to the SE means that there, a SOHO-EIT-detected expanding blob moves away between 13:41 and 14:00 UT (impulsive phase).

evolution of the radio source-hosting loops over a relatively long time. The selected "mean fiber field lines" show some of the expected postflare loop properties. The mean field lines fit reasonably with other imaging information about the plasmamagnetic field structures in AR 8027 (Sect. 4.2). The mean lines evolve almost within a plane which tends slightly more to the vertical position with time. Each mean field line is plotted as seen edge-on onto a least-square adapted plane in Fig. 11a. The lower the mean field line - earlier in the postflare loop evolution - the clearer is the (projected) sigmoidal shape of the line. It is a characteristic property of flares in active regions with sigmoidal loops that the preflare sigmoid is highly sheared, with much of it running along the neutral line. When the sigmoid erupts it leads to the formation of potential (or near-potential) postflare loop arcades normal to the neutral line that appear to expand in time (see e.g. Aurass et al. 1999). In the given case, the mean field line turns like a reverse "S" in contrast to the winding sense of the preflare soft X-ray sigmoid (see e.g. Sterling et al. 2000, Fig. 7c). The sketch in Fig. 11b relates the preflare soft X-ray sigmoid with the fiber burst-detected mean "potential" field line. This relation becomes clear assuming that the fiber burst loops and those loops shaping the NW turn of the soft X-ray preflare sigmoid belong to the same fan. We discussed this point in Sect. 4.2. The SE branch of the preflare soft X-ray pattern, where in the impulsive phase an SOHO-EIT-detected blob is ejected, develops independently from the reformed NW branch what is demonstrated e.g. in Sterling \& Hudson (1997). Our results give an insight into the reconfiguration of the magnetic field during the flare. This will be considered in a forthcoming paper.

Figure 12 shows the path of the single fiber burst potential field line footpoints across the SOHO-MDI magnetogram of the photosphere. The footpoint expansion of the mean field lines is on average about $(7.5 \pm 3.0) \mathrm{km} \mathrm{s}^{-1}$ which agrees well with the $8 \mathrm{~km} \mathrm{~s}^{-1}$ measured for EUV and $12 \mathrm{~km} \mathrm{~s}^{-1}$ for soft X-ray loop footpoint expansion speeds (Sterling et al. 2000, p. 643). The vertical rise speed derived from the field lines

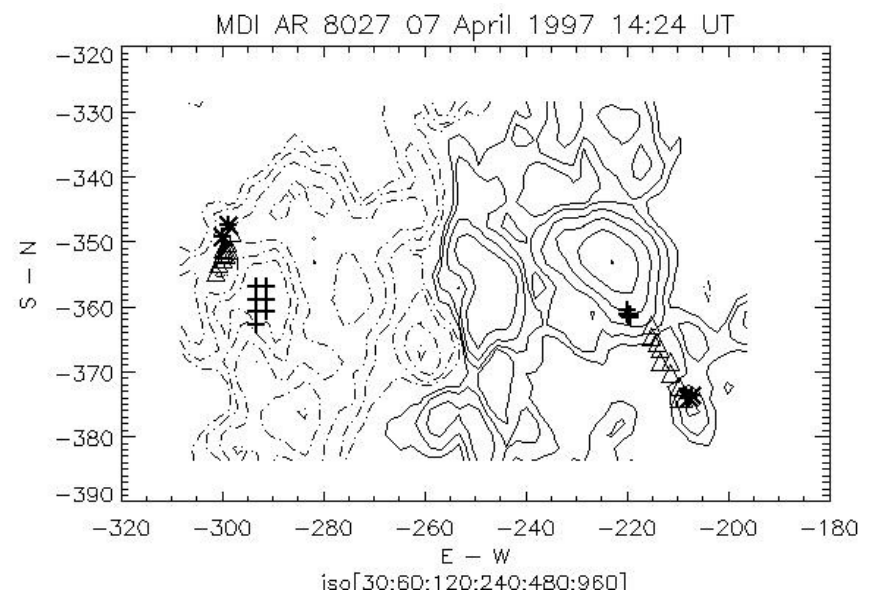

Fig. 12. The further enlarged SOHO-MDI magnetogram from Fig. 4 and the footpoint scatterplot of the selected field lines according to Table 3. Axis in arcsec on the disc, isoline levels in Gauss. “+”-intervals 1 and 2; triangels-interval 5; “*”-interval 6.

cannot be compared to independent observations. There is no other data source available for this height range and on-thedisc-observations. The almost vertical plane revealed by the fiber bursts for the postflare loops is in accordance ${ }^{13}$ with the related observation of the dominantly NW- and SE-propagating coronal wave fronts seen in EIT and the flare-associated halo CME (see Thompson et al. 1999, for wave and CME data). This plane is perpendicular to the preflare sigmoid length axis (e.g. Sterling et al. 2000). We note that the reported acceleration of the fiber burst loop top during the flare is somewhat unexpected and in contrast to the prediction of the rising flux-rope model (e.g. Forbes 1986).

An important result of this work is that the fiber burst source site evolution over time during the EOI reveals a very specific field-related behaviour - the observed source site changes in accordance with a change of the magnetic "weight" between the loop branches. This is predicted by the mechanism of whistler wave excitation in an asymmetric loop (see Sect. 3.1). It reveals that the fiber burst sources experience the footpoint change of the radio source-carrying loops (see Fig. 12), and that the magnetic field in these loops is well represented by the selected potential field lines. Therefore our observations prove the physical significance of the calculated mean "potential" field lines as a good approach to the real field parameters in the considered part of the postflare loop system. It is observed that the fiber burst sources are situated in the same height range (20-100 Mm) but in lower magnetic fields later in the event. If the fiber bursts probe developing postflare loops, these are compatible with the potential field extrapolation of the photopheric magnetic field.

\section{Conclusions}

We present and apply a new method of measuring coronal magnetic fields in postflare loops. To do this we combine AIP radio

${ }^{13}$ In contrast to the strongly inclined soft X-ray cusp-like feature. 
spectral, NRH radio imaging, and SOHO-MDI photospheric magnetic field measurements. We derive a field strength estimate in the corona from the drift rate and the emissionabsorption separation of intermediate drift (fiber) bursts occuring during some type IV radio continua. We presume that the fiber burst exciters are ascending whistler waves in coronal loops driven by trapped nonthermal flare electrons. The radio spectra, combined with radio imaging, give fiber burst position information at least at two frequencies (height levels, assuming a Newkirk (1961) density model). Comparing the source site at two levels with the set of coronal magnetic field lines obtained by potential field extrapolation of the photospheric data yields those parameters of the density model that give the best coincidence between the potential field strength and the fiber burst-derived field strength at two height levels. Furthermore, we obtain a sensitive selection of those potential field lines that cross the fiber burst source volume. With preknowledge about whistler wave excitation in magnetic traps we prove that these field behaviour is physically experienced in the radio sources. This proof is further supported by the comparison of the selected field structures with Yohkoh soft X-ray and SOHO-EUV images. The footpoint spreading of the fiber burst potential field lines coincides with soft X-ray and EUV loop footpoint spread data of other authors. We discuss some new results relevant to fiber burst models and postflare loop dynamics. Our method can be used for coronal magnetic field sounding during flares with the forthcoming new-generation Frequency Agile Solar Radiotelescope (FASR, e.g. Bastian 2003) if the frequency range is extended down to at least $200 \mathrm{MHz}$.

Acknowledgements. The Yohkoh soft X-ray telescope (SXT) image was obtained from the homepage of this cooperative Japanese-USAmerican-UK space mission. The Solar and Heliospheric Observatory (SOHO) is a common activity of ESA and NASA, the Michelson Doppler Imager (MDI) magnetogram data were available for public use at the http://soi.stanford.edu/magnetic/index5.html webpage. The authors are grateful to K.-L. Klein, A. Kerdraon, and G. Trottet for support in access and handling of Nançay Multifrequency Radio Heliograph data. H.A. acknowledges the access to Kanzelhöhe $\mathrm{H}_{\alpha}$ observations (W. Otruba, A. Warmuth). G. Rausche is supported by Grant No. AU 106/13-1 of the Deutsche Forschungsgemeinschaft. The AIP acknowledges the European Office for Aerospace Research and Development for its support in maintaining the solar radio spectral observations at Potsdam. The authors are grateful to the unknown referee for a careful reading and the useful comments.

\section{References}

Aurass, H., Vršnak B., Hofmann, A., \& Rudžjak, V. 1999, Sol. Phys., 190,267

Aurass, H., Vršnak, B., \& Mann, G. 2002, A\&A, 384, 273

Aurass, H., Klein, K.-L., Zlotnik, E.Ya., \& Zaitsev, V. V. 2003, A\&A, 410, 1001

Bastian, T. S. 2003, Proc. SPIE, 4853, 98

Benz, A. O., \& Mann, G. 1998, A\&A, 333, 1034

Bernold, T. 1983, Thesis, ETH Zürich

Bernold, T., \& Treumann, R. 1983, ApJ, 264, 677

Delaboudinière, J.-P., Artzner, G. E., Brunaud, J., et al. 1995, Sol. Phys., 162, 291

Forbes, T. 1986, ApJ, 305, 553

Kosugi, T., Makishima, K., Murakami, T., et al. 1991, Sol. Phys., 136, 17

Krüger, A., \& Hildebrandt, J. 1993, in Proc. IAU Coll., 141, ed. H. Zirin, G. Ai, \& H. Wang, ASP, 46, 249

Kuijpers, J. 1975, Sol. Phys., 44, 173

Mann, G., Karlický, M., \& Motschmann, U. 1987, Sol. Phys., 110, 381

Mann, G., Baumgärtel, K., Chernov, G. P., \& Karlický, M. 1989, Sol. Phys., 120, 383

Mann, G., Jansen, F., MacDowall, R. J., Kaiser, M. L., \& Stone, R. G. 1999, A\&A, 348, 614

Martens, P. C. H., Kuin, N. P. M. 1989, Sol. Phys., 122, 263

McLean, D. J., \& Labrum, N. R. 1985, Sol. Radiophysics (Cambridge: Univ. Press)

Newkirk, G. A. 1961, ApJ, 133, 983

Priest, E. R., \& Forbes, T. G. 1990, Sol. Phys., 126, 319

Priest, E. R., \& Forbes, T. G. 2000, Magnetic Reconnection (Cambridge: Univ. Press)

Sakurai, T. 1982, Sol. Phys., 76, 301

Scherrer, P. H., Bogart, R. S., Bush, R. I., et al. 1995, Sol. Phys., 162 129

Seehafer, N. 1978, Sol. Phys., 58, 215

Seehafer, N., \& Staude, J. 1980, Sol. Phys., 67, 121

Slottje, C. 1981, Atlas of Fine Structures of Dynamic Spectra... (Utrecht: Publ. Utrecht Observatory)

Sterling, A. C., \& Hudson, H. S. 1997, ApJ, 491, L55

Sterling, A. C., \& Moore, R. L. 2004, ApJ, 602, 1024

Sterling, A. C., Hudson, H. S., Thompson, B. J., \& Zarro, D. M. 2000, ApJ, 532, 628

The Radio Heliograph Group 1993, Adv. Sp. Res., 13(9), 411

Thompson, B. J., Gurman, J. B., Neupert, W. M., et al. 1999, ApJ, 517, L151

Treumann, R. A., Güdel, M., \& Benz, A. O. 1990, A\&A, 236, 242

Zarro, D. M., Sterling, A. C., Thompson, B. J., Hudson, H. S., \& Nitta, N. 1999, ApJ, 520, L139

Zlotnik, E. Ya., Zaitsev, V. V., Aurass, H., Mann, G., \& Hofmann, A. 2003, A\&A, 410, 1011 